\title{
Bronchoscopy as a Useful Examination for Determining Surgical Treatment Indications in Refractory Mycobacterium avium Complex Lung Disease Patients with Bilateral Lesions
}

Erina Tabata ${ }^{1}$, Akimasa Sekine ${ }^{1}$, Eri Hagiwara ${ }^{1}$, Michihiko Tajiri ${ }^{2}$ and Takashi Ogura ${ }^{1}$

\begin{abstract}
:
We herein report three cases of refractory Mycobacterium avium complex (MAC) disease successfully treated surgically despite the MAC lesions being present bilaterally. Of note, although two patients did not present with any respiratory symptom, bronchoscopy clearly revealed a major excretory lesion with a large amount of purulent sputum in all patients. Because an excretory lesion was localized, surgical resection was performed, and the mycobacterial sputum smear became negative in all patients. Bronchoscopy may be a useful examination for detecting major excretory lesions with purulent sputum, which can disseminate to other lobes, and for determining the surgical indications of refractory MAC patients, regardless of the presence of respiratory symptoms.
\end{abstract}

Key words: bronchoscopy, surgical resection, Mycobacterium avium complex, bilateral, refractory

(Intern Med 58: 973-978, 2019)

(DOI: 10.2169/internalmedicine.1370-18)

\section{Introduction}

The prevalence of nontuberculous mycobacteria (NTM) lung disease continues to increase worldwide (1). In addition, the morbidity and mortality of NTM lung disease have also increased; therefore, managing NTM patients has become an increasing problem(2). Mycobacterium avium complex (MAC), comprising M. avium and M. intracellulare, is the most common cause of NTM lung disease in Japan. The American Thoracic Society (ATS) and the Infectious Diseases Society of America (IDSA) officially stated in 2007 that the initial therapy of MAC lung disease is a multidrug-regimen comprising clarithromycin (CAM), ethambutol (EB), and rifampin (RFP) (3). However, treatment success rates (approximately 40-44\%) for MAC patients undergoing multidrug chemotherapy are unsatisfactory (4). Therefore, the 2007 ATS/IDSA statement also reported on the outcomes of surgical treatments for MAC disease that is refractory to chemotherapy but predominantly localized to one lung. In addition, the Japanese Society for Tuberculosis declared that excising major excretory NTM lesions is effective for suppressing disease progression (5). Since the release of the ATS/IDSA statement, a number of papers have been published concerning surgical resection of major lesions due to NTM lung disease (6-10). However, there are no widely accepted or uniform criteria for selecting good candidates for surgically treatable MAC.

We herein report three patients with refractory MAC disease who successfully underwent surgical resection although MAC lesions were bilaterally present.

\section{Case Reports}

\section{Case 1}

A 63-year-old woman was diagnosed with MAC disease in 2008 and treated with multidrug chemotherapy comprising CAM, RFP, EB, and kanamycin since 2013. Chest computed tomography (CT) revealed nodules and bronchiectasis

${ }^{1}$ Department of Respiratory Medicine, Kanagawa Cardiovascular and Respiratory Center, Japan and ${ }^{2}$ Department of Thoracic Surgery, Kanagawa Cardiovascular and Respiratory Center, Japan

Received: April 16, 2018; Accepted: August 22, 2018; Advance Publication by J-STAGE: November 19, 2018

Correspondence to Dr. Akimasa Sekine, Akimasa.Sekine@gmail.com 


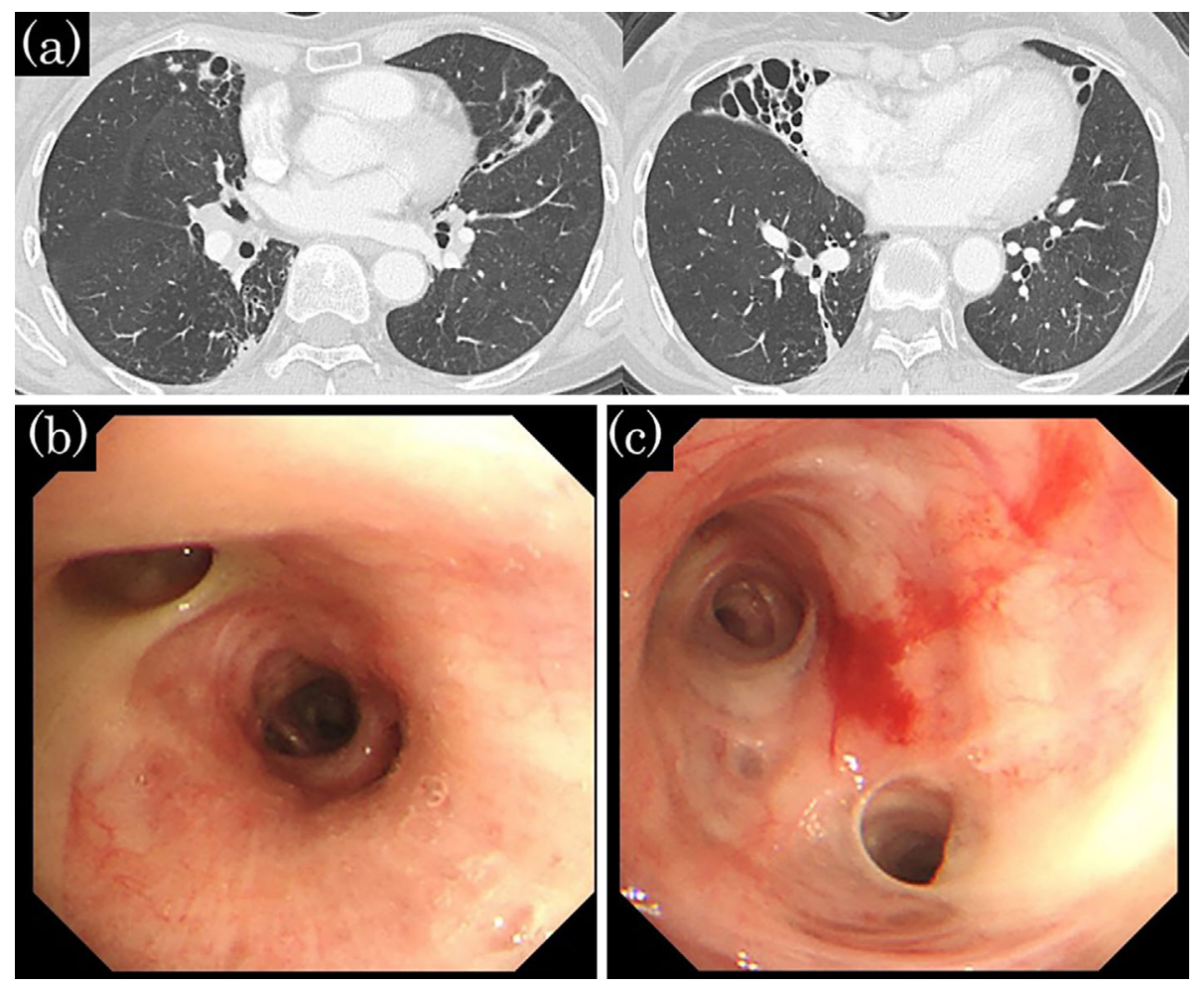

Figure 1. Bronchiectasis bilaterally present in the middle lobe and lingular segment (a). Bronchoscopy shows a large amount of purulent secretion from the right middle lobe bronchus and no inflammatory changes, such as edema or redness of the mucosal surface of the bronchus (b). There was no secretion from the left lingular bronchus with submucosal contact bleeding (c).

bilaterally in the middle lobe and lingular segment (Fig. 1a). Despite two-year treatment, the sputum mycobacterium smear remained positive $(1+)$, although she had no respiratory symptoms. In 2015, bronchoscopy was performed to investigate whether or not the major excretory lesion was localized, and a large amount of purulent secretion from the right middle lobe bronchus was detected (Fig. 1b). However, there was no secretion from the left lingular bronchus, which radiologically corresponded to bronchiectasis (Fig. 1c). Clinically significant bacteria, such as Pseudomonas aeruginosa, were not cultured. These bronchoscopic findings indicated that a major excretory lesion was present on the right middle lobe, so we performed right middle lobectomy. With continuing anti-MAC chemotherapy, her sputum smear was confirmed to be negative at 3 and 12 months after surgery (Table).

\section{Case 2}

A 42-year-old woman with bilateral bronchiectasis in the middle lobe and lingular segment underwent right middle lobectomy in 2012. She underwent multidrug chemotherapy comprising CAM, RFP, EB, and streptomycin two years before and three years after surgery, but her mycobacterium sputum smear remained positive (2+) in 2015. Co-infection was denied based on the findings of a bacteriological examination. CT revealed bronchiectasis and consolidation in the left lingular segment and multiple small centrilobular nodules in both lower lobes (Fig. 2a). Although she felt no ap- parent respiratory symptoms, preoperative bronchoscopy showed a large amount of purulent secretion in the left lingular bronchus (Fig. 2b) with a normal right bronchus intermedius (Fig. 2c). Therefore, she underwent lingulectomy in December 2015 while continuing multidrug chemotherapy. After surgical resection, she detected a decrease in the amount of sputum, suggesting that she had had some sputum before surgery. With continuing anti-MAC chemotherapy, her sputum smear was confirmed to be negative at 3 and 12 months post-surgery (Table), and the radiological centrilobular nodular opacities in both lower lobes disappeared in December 2016.

\section{Case 3}

A 61-year-old woman was diagnosed with MAC lung disease in 1997. Because 2.5-year multiple drug chemotherapy including RFP, EB, and CAM was ineffective (Fig. 3a), resection of the right S2 segment was performed in 2001. Although her condition remained stable for seven years after surgery, the MAC disease in the right lower lobe gradually worsened; in 2012, she re-initiated multidrug chemotherapy. However, CAM turned out to be resistant in 2014, and she subsequently presented with a fever and increased sputum from May 2015. CT revealed a cavitary lesion in the right upper lobe and widespread consolidation in the right middle and lower lobes. In addition, some nodules with bronchiectasis were observed in the left lingular segment and lower lobe (Fig. 3b-d). Because the consolidation rapidly aggra- 
Table. Summary of Surgical Treatment Outcome in Our Three Patients.

\begin{tabular}{|c|c|c|c|}
\hline & Case 1 & Case 2 & Case 3 \\
\hline Age (years)/Sex & $63 / \mathrm{F}$ & $42 / \mathrm{F}$ & $61 / \mathrm{F}$ \\
\hline Duration of MAC disease & 7 years & 5 years & 18 years \\
\hline \multirow[t]{4}{*}{ Treatment regimen (mg/body/day) } & RFP $300 \mathrm{mg}$ & RFP $300 \mathrm{mg}$ & RFP $450 \mathrm{mg}$ \\
\hline & EB $500 \mathrm{mg}$ & EB $500 \mathrm{mg}$ & EB $500 \mathrm{mg}$ \\
\hline & CAM $800 \mathrm{mg}$ & CAM $800 \mathrm{mg}$ & $\mathrm{KM} 1,000 \mathrm{mg}$ \\
\hline & KM $500 \mathrm{mg}$ & & STFX $100 \mathrm{mg}$ \\
\hline Resected excretory lesion & Rt middle lobectomy & Lt upper lobectomy & Rt pneumonectomy \\
\hline \multicolumn{4}{|l|}{ Respiratory symptoms } \\
\hline before surgery & None & None $\S$ & Sputum, cough \\
\hline after surgery & None & Improved $^{\S}$ & Improved \\
\hline \multicolumn{4}{|l|}{ Mycobacterial sputum smear/culture } \\
\hline before surgery & $1+/+$ & $2+/+^{*}$ & $2+/+$ \\
\hline \multicolumn{4}{|l|}{ after surgery } \\
\hline 3 months & $-/+^{*}$ & $-/+^{*}$ & $-/-$ \\
\hline 12 months & $-/-^{*}$ & $-I^{*}$ & $-/-$ \\
\hline
\end{tabular}

F: female, MAC: mycobacterium avium complex, lt: left, rt: right

$\S$ Despite experiencing no respiratory symptoms at surgical resection, the patient noticed that she had had some sputum before surgical resection.

*induced sputum

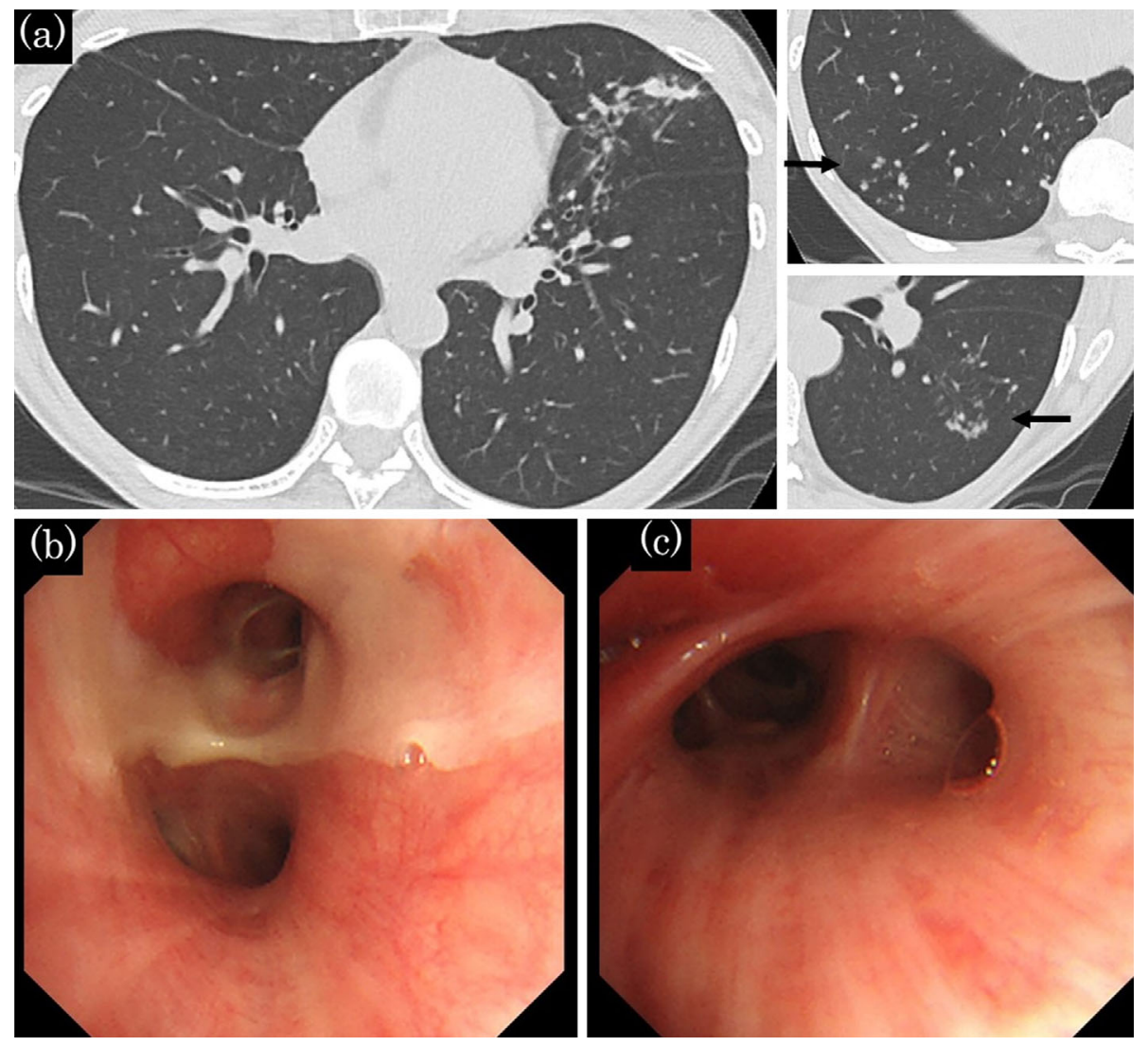

Figure 2. Chest computed tomography revealed bronchiectasis and consolidation in the left lingular segment and multiple small centrilobular nodular opacities (arrow) in both lower lobes (a). Preoperative bronchoscopy showed a large amount of purulent secretion in the left lingular bronchus (b) with a normal right bronchus intermedius (c).

vated and a mycobacterium sputum smear remained positive (2+) despite multidrug chemotherapy comprising RFP, EB, kanamycin, and sitafloxacin, we performed bronchoscopy to investigate whether the major excretory lesion was extensive or localized. Bronchoscopy revealed a large amount of purulent secretion in the right intermediate bronchus and the right B2 bronchial stump (Fig. 4a and b), suggesting a potential bronchopleural fistula. No apparent purulent secretion 


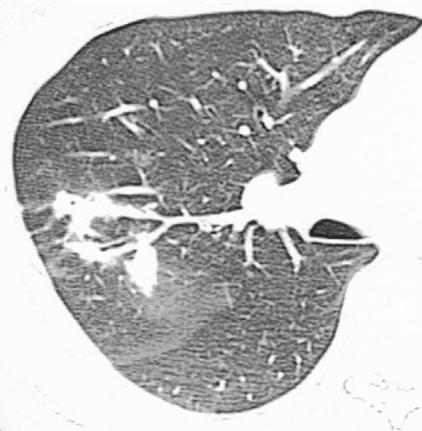

(a)

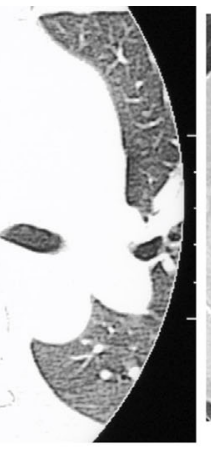

.

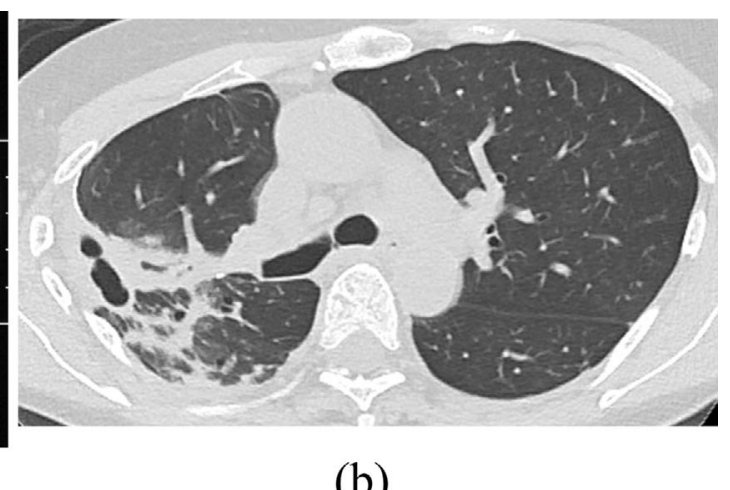

(b)

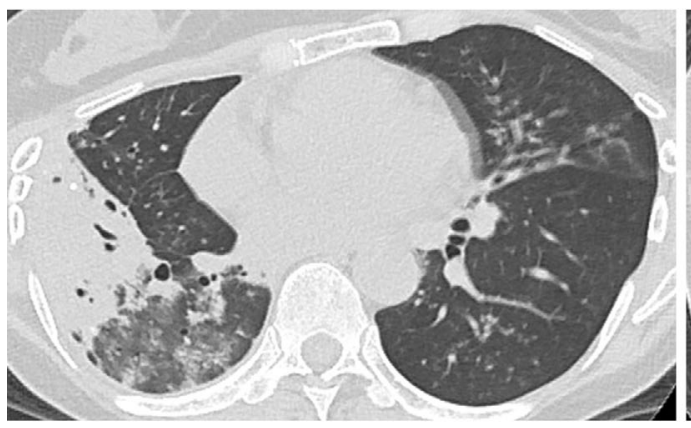

(c)

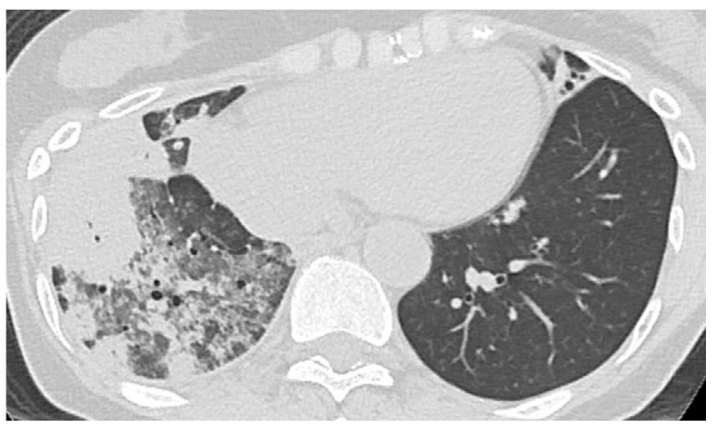

(d)

Figure 3. (a) Consolidation with bronchiectasis was observed in the right upper lobe. (b) A cavitary lesion was observed in the right remaining upper lobe. (c-d) Widespread consolidation was evident in the right lung, and some nodules with bronchiectasis were observed in the left lingular segment and lower lobe.

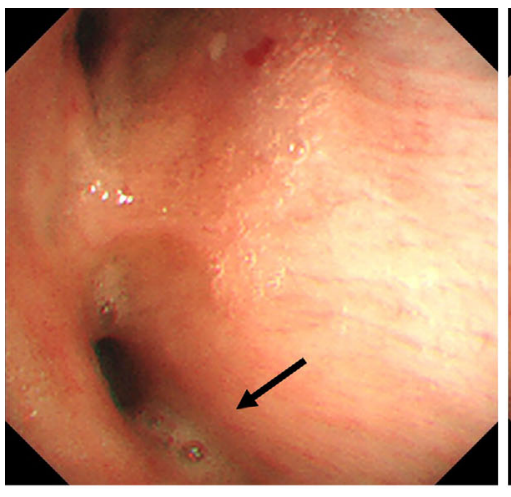

(a)

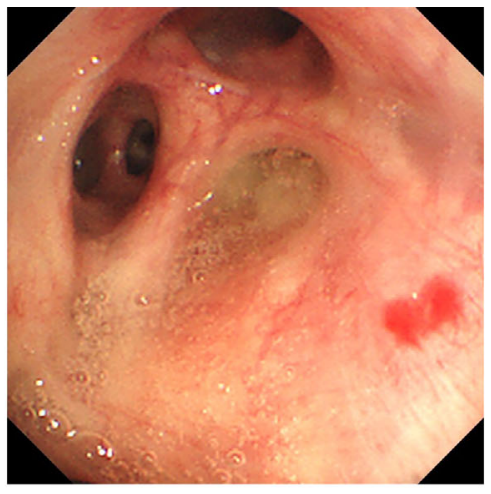

(b)

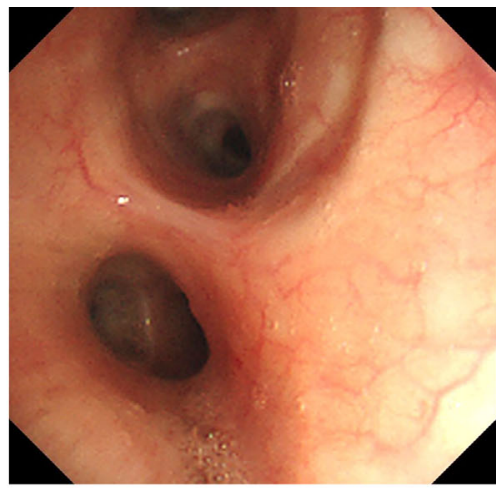

(c)

Figure 4. Preoperative bronchoscopy showed a large amount of purulent secretion in the right bronchus intermedius (a) (arrow) and B2 bronchial stump (b), although no purulent excretion was observed around the left second carina (c).

was observed in any left bronchi (Fig. 4c), and no bacteria other than MAC were detected. Because a pulmonary perfusion test showed a drastic decrease in the right lung compared to left one (16\% vs. $84 \%$ ), she underwent right pneumonectomy with bronchial stump reinforcement with muscle flaps in August 2015. Her subjective symptoms drastically improved immediately after surgery, and home-oxygen therapy was unnecessary. Anti-MAC chemotherapy was continued, and her sputum smear was confirmed to be negative at
3 and 12 months after surgical resection (Table).

\section{Discussion}

We encountered three cases of refractory MAC disease that were successfully surgically resected, although the MAC lesions were radiologically present in bilateral lungs. Our three cases are summarized in Table. Although the follow-up period was not very long and therefore careful ob- 
servation is needed, this study provides two clinical implications regarding bronchoscopic efficacy.

First, bronchoscopy can provide important information about the presence of a major excretory lesion with purulent sputum, even for asymptomatic patients. Generally, MAC lung disease gradually progresses along with dissemination from excretory lesions to other lobes via the airway (11). In fact, although the degree of bronchiectasis in the lingular division was not severe in any of our patients, especially in Case 2, a large amount of purulent excretion was present, suggesting potential dissemination to other lobes. Therefore, it is crucial to confirm whether or not an excretory lesion is present. Of note, although two of our patients (Cases 1 and 2) did not complain of any respiratory symptoms, bronchoscopy revealed a large amount of purulent sputum in all three patients. After surgical resection, one patient (Case 2) noticed that she had had sputum before surgery. Because it is not easy for respiratory physician to decide on surgical indications for patients with refractory but asymptomatic MAC disease, bronchoscopy can help prevent any underestimation of the disease activity, which may lead to appropriate surgical treatment.

Second, bronchoscopy can aid in directly confirming where or not the major excretory lesion is localized, especially in MAC patients with radiologically bilateral lesions. Although we were unable to analyze the bronchial lavage fluid to identify mycobacteria at the opposite site, the findings of a bronchoscopic examination suggested that the purulent excretion was localized. To date, a number of papers have been published concerning NTM lung disease treated with surgical resection (6-10). However, most of those papers failed to provide detailed information about whether the NTM lesions were unilateral or bilateral. Only five reported cases of non-localized bilateral NTM lung disease were successfully treated with two-stage bilateral resection: all five patients underwent right middle lobectomy and subsequent left lingulectomy (6). In addition, there was one case of bilateral MAC disease that was successfully treated with single lobectomy in combination with chemotherapy (12). However, these papers did not mention performing bronchoscopy when deciding on the indication of surgical treatment. In general, in clinical practice, major excretory lesions are evaluated only with CT and not with bronchoscopy. Of note, previous reports have revealed that the postoperative complication rate, including bronchopleural fistula, is relatively high (11.7-24\%) in NTM patients (6-10); it is therefore important to minimize the extent of surgical lung resection. Our results strongly suggest that bronchoscopy can clarify whether or not major excretory lesions are localized; purulent excretion was limited to the bronchus of one lobe, and sputum smear conversion was obtained after surgically resecting the major excretory lesion, as shown in Table. In addition, we also previously reported that intrabronchial secretion can be a manifestation of ongoing airway damage, indicating the need for further treatment (13). Taken together, these present and previous findings suggest that broncho- scopy should be considered for investigating whether or not intrabronchial secretion is localized in refractory MAC patients with bilateral lesions.

\section{Conclusion}

We herein reported three refractory MAC patients with bilateral radiological lesions who were successfully treated by surgery. Bronchoscopy may be a useful examination for detecting major excretory lesions with purulent sputum, which can disseminate to other lobes, and determining surgical treatment indications for refractory MAC patients, regardless of the presence of respiratory symptoms. Because our case series was too small to reach a definitive conclusion, it is necessary to accumulate more cases in which an operation is performed on the basis of bronchoscopy examination findings.

Appropriate written informed consent was obtained for publication of this case report and accompanying images.

The authors state that they have no Conflict of Interest (COI).

\section{References}

1. Egelund EF, Fennelly KP, Peloquin CA. Medications and monitoring in nontuberculous mycobacteria infections. Clin Chest Med 36: 55-66, 2015.

2. Morimoto K, Iwai K, Uchimura $\mathrm{K}$, et al. A steady increase in nontuberculous mycobacteriosis mortality and estimated prevalence in Japan. Ann Am Thorac Soc 11: 1-8, 2014.

3. Griffith DE, Aksamit T, Brown-Elliott BA, et al. An official ATS/ IDSA statement: diagnosis, treatment, and prevention of nontuberculous mycobacterial diseases. Am J Respir Crit Care Med 175: 367-416, 2007.

4. Xu HB, Jiang RH, Li L. Treatment outcomes for Mycobacterium avium complex: a systematic review and meta-analysis. Eur J Clin Microbiol Infect Dis 33: 347-358, 2014.

5. Committee on the Management of Non-Tuberculous Acid-Fast Bacterial Infections of the Lung, the Japanese Society for Tuberculosis. Guideline for surgical therapy of non-tuberculous acid-fast bacterial infection of the lung. Kekkaku 83: 527-528, 2008 (in Japanese).

6. Shiraishi Y, Katsuragi N, Kita H, Hyogotani A, Saito MH, Shimoda K. Adjuvant surgical treatment of nontuberculous mycobacterial lung disease. Ann Thorac Surg 96: 287-291, 2013.

7. Kang HK, Park HY, Kim D, et al. Treatment outcomes of adjuvant resectional surgery for nontuberculous mycobacterial lung disease. BMC Infect Dis 15: 76, 2015.

8. Asakura T, Hayakawa N, Hasegawa N, et al. Long-term outcome of pulmonary resection for nontuberculous mycobacterial pulmonary disease. Clin Infect Dis 65: 244-251, 2017.

9. Sakane T, Matsuoka K, Kumata S, et al. The outcomes of anatomical lung resection for nontuberculous mycobacterial lung disease. J Thorac Dis 10: 954-962, 2018.

10. Mitchell JD, Bishop A, Cafaro A, Weyant MJ, Pomerantz M. Anatomic lung resection for nontuberculous mycobacterial disease. Ann Thorac Surg 85: 1887-1892, 2008.

11. Fujita J, Ohtsuki Y, Suemitsu I, et al. Pathological and radiological changes in resected lung specimens in Mycobacterium avium intracellulare complex disease. Eur Respir J 13: 535-540, 1999.

12. Hamada N, Kawata N, Shibayama T, et al. Non-localized Mycobacterium avium lung disease successfully treated with lobectomy 
and chemotherapy. Intern Med 49: 1233-1236, 2010.

13. Sekine A, Saito T, Satoh H, Morishita Y, Tsunoda Y. Limited value of transbronchial lung biopsy for diagnosing Mycobacterium avium complex lung disease. Clin Respir J 11: 1018-1023, 2017.
The Internal Medicine is an Open Access journal distributed under the Creative Commons Attribution-NonCommercial-NoDerivatives 4.0 International License. To view the details of this license, please visit (https://creativecommons.org/licenses/ by-nc-nd/4.0/).

(C) 2019 The Japanese Society of Internal Medicine Intern Med 58: 973-978, 2019 